\title{
Ultrasonic Flaw Detection in Composite Materials Using SSP-MPSD Algorithm
}

\author{
BENAMMAR Abdessalem ${ }^{\dagger}$ and DRAI Redouane*
}

\begin{abstract}
Due to the inherent inhomogeneous and anisotropy nature of the composite materials, the detection of internal defects in these materials with non-destructive techniques is an important requirement both for quality checks during the production phase and in service inspection during maintenance operations. The estimation of the time-of-arrival (TOA) and/or time-of-flight (TOF) of the ultrasonic echoes is essential in ultrasonic non-destructive testing (NDT). In this paper, we used split-spectrum processing (SSP) combined with matching pursuit signal decomposition (MPSD) to develop a dedicated ultrasonic detection system. SSP algorithm is used for Signal-to-Noise Ratio (SNR) enhancement, and the MPSD algorithm is used to decompose backscattered signals into a linear expansion of chirplet echoes and estimate the chirplet parameters. Therefore, the combination of SSP and MPSD (SSP-MPSD) presents a powerful technique for ultrasonic NDT. The SSP algorithm is achieved by using Gaussian band pass filters. Then, MPSD algorithm uses the Maximum Likelihood Estimation. The good performance of the proposed method is experimentally verified using ultrasonic traces acquired from three specimens of carbon fibre reinforced polymer multi-layered composite materials (CFRP).
\end{abstract}

Keywords: Non-Destructive testing, Ultrasonics, Carbon fibre, Defects, Signal processing

\section{Introduction}

In recent years, due to their great potential in weight saving, fiber reinforced laminated composites are becoming increasingly important in applications where low weight, high stiffness, and high strength are required, in particular in the aircraft industry. Composite structures can be damaged under mechanical and thermal loadings. The development of an effective technique to monitor and detect the size, location and orientation of any internal defects may serve to help to avoid composite failures.

Currently, new airplanes are manufactured with carbon fiber composite materials to be lighter and more fuel efficient. With the increased usage of fiber reinforced materials in structural materials, it has become all the more important for the NDT community to develop viable technique which can be used to test these materials while they are in service. Typical NDT techniques which have been used to try and reconstruct signals and / or images of defects inside the metals and composite materials are visual optical inspection, radiography, magnet particle inspection, eddy current testing, ultrasonic testing, acoustic emission testing, microwave testing, etc. [1-2]

The ultrasonic testing is based on the detection and the interpretation of the ultrasonic waves reflected by defects.

\footnotetext{
$\dagger$ Corresponding Author: Image and Signal Processing Laboratory, Welding \& NDT Research Center (CSC), Route de Dely-Ibrahim, BP 64, Cheraga, Algeria. (Abs_benammar@yahoo.fr)

* Welding \& NDT Research Center (CSC), Route de Dely-Ibrahim, BP 64, Cheraga, Algeria.

Received: October 27, 2013; Accepted: April 14, 2014
}

Widely used methods at the present time are split spectrum processing [3-7], wavelet method [8-16], the high resolution pursuit (HRP) [17-18], and the chirplet transform [1920]. The results are presented in various forms, so a direct comparison is very difficult.

In this paper, a new approach to the inspection problem of composite materials using ultrasonic signal processing is proposed in order to detect delamination defects and thicknesses measurement. We consider two main steps for interpreting ultrasonic data: the pre-processing technique necessary to enhance the SNR, this step is realized by SSP algorithm. The second step was carried out by using matching pursuit signal decomposition (MPSD), this technique is necessary for the estimation of the time-of-arrival (TOA) and/or time-of-flight (TOF) of the ultrasonic echoes.

This paper is organized as follows: Section 2 describes the proposed method for flaw detection using SSP-MPSD system structure. In section 3, the performance of the algorithm is tested and compared using simulated ultrasonic echoes. In section 4 the performance of the method is experimentally verified using ultrasonic traces acquired from three specimens of carbon fibre reinforced polymer multi-layered composite materials (CFRP) provided by an aircraft manufacturer company. Finally, conclusions are given in Section 5.

\section{Proposed Method for Flaw Detection}

In this work, a new approach is presented for ultrasonic 
flaw detection applications. This approach is based on split-spectrum processing (SSP) combined with matching pursuit signal decomposition (MPSD).

\subsection{Ultrasound signal formulation}

The main aim of the analysis of the signal received by ultrasonic receiver in an NDT process is to obtain information about the material integrity.

We therefore need to detect a possible reflection induced by a defect in the structure in a signal that is corrupted by the structural noise generated mainly by the reinforcement, such as carbon fibers, in the matrix material. From a mathematical point of view, we can define the problem as follows:

$$
\begin{gathered}
x(t)=y(t)+n(t)=\sum_{m=1}^{M} s\left(t ; \Theta_{m}\right)+n(t) \\
x(t)=\beta_{m} \exp \left(-\alpha_{1 m}\left(t-\tau_{m}\right)^{2}\right) \times \\
\cos \left[-\alpha_{2 m}\left(t-\tau_{m}\right)^{2}+2 \pi f_{c m}\left(t-\tau_{m}\right)+\varphi_{m}\right]+n(t)
\end{gathered}
$$

In this equation, $x(t)$ represents the observed noisy signal, $y(t)$ is the model-based noiseless signal and $n(t)$ represents the electrical and structural noise. The parameter vector

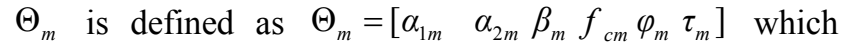
contains all the unknown parameters of the problem, the bandwidth factor $\alpha_{1 m}$, the chirp rate $\alpha_{2 m}$, the amplitude $\beta_{m}$, the center frequency $f_{c m}$, the phase $\varphi_{m}$, and the arrival time $\tau_{m}$.

\subsection{The structure of SSP-MPSD}

The overall system diagram of SSP-MPSD is given in Fig. 1, including a pre-process (i.e., SSP) and post-process (i.e., MPSD). SSP technique [4] splits the received wide band signal into a group of diverse frequency narrow band signals exhibiting different SNR, and subsequently recombine those using non linear techniques in order to increase this SNR. The principle of this technique is illustrated in Fig. 1. The performance of the SSP is sensitive to four parameters which are the number of filters used for splitting spectrum, the filter band width, the step frequency between filters and the position of passband filter (the frequency center of the first and the last filter) [21].

The reconstruction stage of SSP output may be achieved by several non linear algorithms to improve SNR of the input signal. In this work, we use the arithmetic mean [22] defined as:

$$
z(t)=\frac{1}{N} \sum_{i=1}^{N} x_{i}(t)
$$

Where $x_{i}(t) i=1,2, \ldots, N$ represent the narrowband output signals. The arithmetic mean essentially acts as a band pass

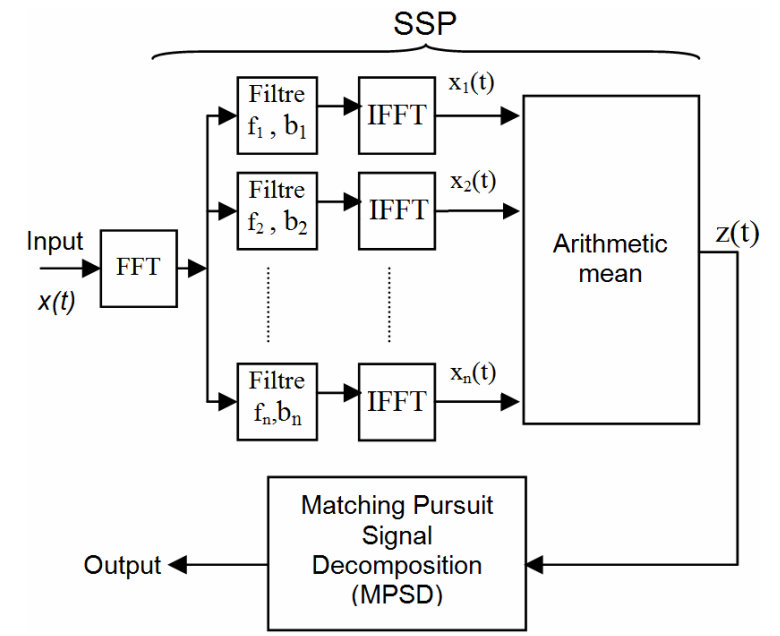

Fig. 1. The structure of SSP-MPSD

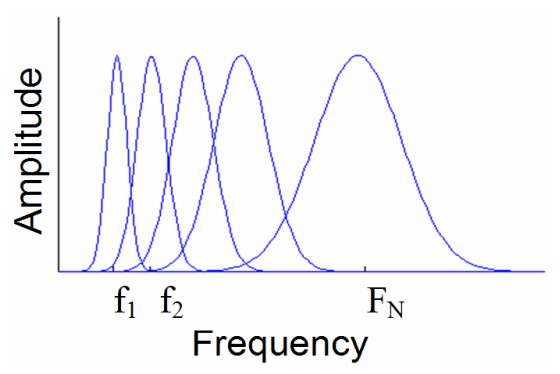

Fig. 2. Frequency response of the constant- $Q$ filter bank $Q$ is the quality factor defined as $Q=f_{i} / b_{i}$, with $f_{i}$ the center frequency and $b_{i}$ the band width of filters.

filter since it linearly combines the sampled waveforms from different frequency regions of the input wide band signal.

In this work, we use the constant- $Q$ decomposition. SSP algorithm gives better results when the decomposition is achieved by constant- $Q$ filters as shown in [23] (see Fig. 2).

In this work the SSP described above presents only signal features and can be viewed as an initial stage of signal processing. While SSP can provide signal features, we still need another algorithm to detect the echoes, so the MPSD has been introduced for this aim. MPSD algorithm has been developed based on the matching pursuit signal decomposition and the Maximum Likelihood Estimation [20]. The matching pursuit (MP) algorithm is an adaptive signal decomposition technique that is energy conservative [24]. The MP algorithm first matches a function to the original signal. Then, this best matching function is subtracted from the signal to obtain the signal residue. At each iteration a new function is matched to the current signal residue. When the energy of signal residue is a fraction of the energy of the original signal the decomposition is completed. The final decomposition is a linear expansion of all chosen matching functions. In the original matching pursuit algorithm, the best match criterion uses correlation criteria to determine the best matching 
function. The real challenge of matching pursuit signal decomposition is that different matching criteria can produce different decomposition results [24-25]. In the optimization stage of the matching pursuit signal decomposition (MPSD) algorithm, the signal residue is represented by a chirplet function and the remaining signal (i.e. next residue),

$$
R^{n} s=g(t ; \Theta)+R^{n+1} s
$$

Here, $R^{n} s$ is the current signal residue $z(t), R^{n+1} s$ is the next signal residue and $g(t ; \Theta)$ is a chirplet echo

$$
\begin{aligned}
& g(t ; \Theta)=\beta e^{-\alpha_{1}(t-\tau)^{2}} \times \\
& \left.\cos \left[\alpha_{2}(t-\tau)^{2}+2 \pi f_{c}(t-\tau)+\phi\right)\right]
\end{aligned}
$$

Where $\Theta=\left[\begin{array}{llllll}\alpha_{1} & \alpha_{2} & \beta & f_{c} & \phi & \tau\end{array}\right]$ denotes the parameter vector of $g(t ; \Theta)$. If we assume $R^{n+1} s$ has white Gaussian noise characteristics, the MLE of the parameter vector $\Theta$ can be obtained by minimizing:

$$
\Theta_{M L E}=\arg _{\Theta} \min \left\|R^{n} s-g_{\Theta}(t)\right\|^{2}
$$

In summary, the iterative MPSD algorithm can be briefly stated as follows [20]:

(1) Initialization: Set iteration index $n=0$ and first signal residue $R^{n} s=z(t)$.

(2) Find the best parameter vector of the chirplet function: $\Theta_{M L E}=\arg _{\Theta} \min \left\|R^{n} s-g_{\Theta}(t)\right\|^{2}$, and set $g_{n}=g\left(t ; \Theta_{n}\right)$.

(3) Update: Compute the next residue $R^{n+1} s=R^{n} s-g_{n}$

(4) Check convergence: If $\frac{\left\|R^{n+1} s\right\|^{2}}{\|s(t)\|^{2}} \leq$ Threshold, stop the iterative procedure; else; $n=n+1$ and go to Step 2. Jump to step 2 and go on.

In the next section we applied both simulated and experimental ultrasonic signals to test SSP-MPSD algorithm, and show its performance.

\section{Simulation Study}

In this section we evaluate the robustness of the proposed SSP-MPSD algorithm compared to our previous work [7]. For easy comparison with previous published results, we have used the same ultrasonic synthetic signal with different levels of signal to noise ratio. We simulated ultrasonic signal with two echoes. The frequency is supposed to be centered at $2.25 \mathrm{Mhz}$. The parameter $\Delta \tau$ or the time difference of arrival (TDOA) of ultrasonic echoes has then been modified, varying from $0.1 \mu \mathrm{s}$ to $1 \mu \mathrm{s}$. The
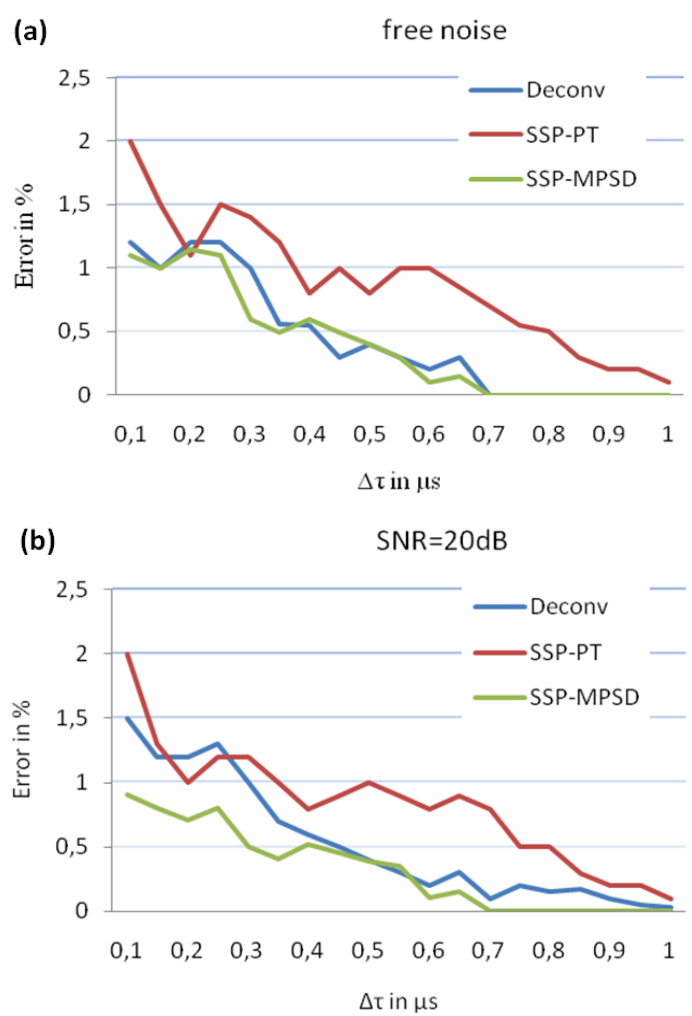

Fig. 3. (a) Error $\Delta \tau$ measurement with noise less signal; (b) Error $\Delta \tau$ measurement with $\mathrm{SNR}=20 \mathrm{~dB}$

SSP-MPSD, SSP-PT and deconvolution by EM algorithm have been applied for estimating $\Delta \tau$ and the backscattered signal has been reconstructed. Fig. 3 shows the error (in \%), between the simulated and the reconstructed signal, as a function of the distance $\Delta \tau$.

In Figs. 3(a) and Fig. 3(b), we can note that the noise level does not have a large influence on the SSP-PT, but this method does not give a good detection. The results obtained by the deconvolution method are very similar to the SSP-MPSD method in the case of noiseless signal. In the case of noisy signal the detection efficiency of SSPMPSD is better than that of the deconvolution and the SSPPT. Based on these simulations; the best results were obtained with the SSP-MPSD method.

\section{Experimental Results and Discussion}

Experimental tests were performed on three specimens of carbon fiber reinforced polymer multi-layered composite materials (CFRP), provided by an aircraft manufacturer company. Typically, delaminations in thin composite laminates are detectable by an immersion transducer operating in pulse-echo mode. Fig. 4 shows schematic diagram of the experimental system. The three samples contain three different types of delamination defects. Multiple signals are reflected from the surfaces of the specimen as well as from delaminations, as shown in Fig. 4. 


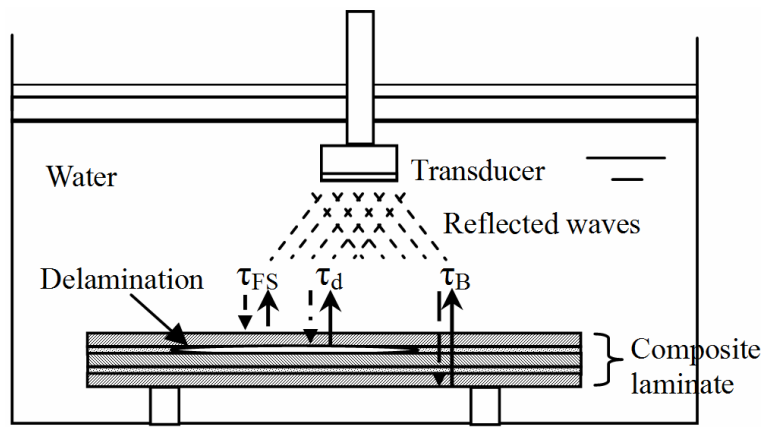

Fig. 4. Schematic diagram of the experimental system

The thickness and the delamination depth in the material can be given using the equation:

$$
d=\mid V_{\text {sample }}\left(\tau_{d, B}-\tau_{F S}\right\rfloor / 2
$$

where, $V_{\text {sample }}$ is the velocity of ultrasound in the material; $\tau_{F S}$ represents the time location of the ultrasonic front surface echo; $\tau_{B}$ represents the time location of the ultrasonic first back wall echo of the sample; $\tau_{d}$ represents the defect echo location of the delamination zone; $\left(\tau_{d, B}-\right.$ $\tau_{F S}$ ) represents the pulse-echo time delay between a front surface and back wall echoes or delamination echoes.

The signals are obtained using tow transducers centered at frequency $2.25 \mathrm{Mhz}$ and $5 \mathrm{Mhz}$, with quality factor 1.8 and 1.92, respectively. Longitudinal waves are used, the sound velocity in this material is $V_{\text {sample }}=2830 \mathrm{~m} / \mathrm{s}$.

The specimen (A) is made of eight layers joined with epoxy, one layer on the other altering the orientation from $\left(0^{\circ}, 45^{\circ}\right)$. This specimen is divided in two zones (Fig. 5):

- The $1^{\text {st }}$ zone is an undamaged zone with $2.7 \mathrm{~mm}$ thickness.

- The $2^{\text {nd }}$ zone is a damaged zone. The delamination defect is a Teflon blade, with $0.6 \mathrm{~mm}$ thickness, situated before the end of the last layer (i.e. between layers 7 and 8).

Fig. 6 (a) shows the measured echoes reflected from the front and back surfaces of CFRP specimen (A) in the undamaged zone with $2.25 \mathrm{Mhz}$ transducer. Three echoes can be found in the signal: The first echo represents the front surface echo, the second echo represents the first back

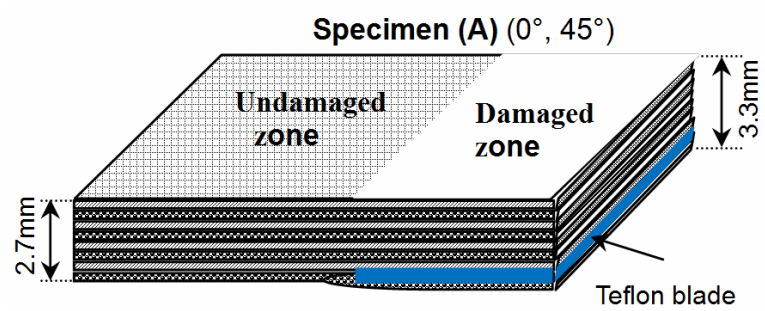

Fig. 5. Geometry and dimension of the CFRP specimen (A) (delamination defect is a Teflon blade situated between layers 7 and 8 ) wall echo and the third echo represents the second back wall echo. The results obtained by the three methods are shown in Fig. 6 (b). The time difference of arrival (TDOA) obtained by deconvolution $\Delta \tau_{\text {deconv }}=1.89 \mu \mathrm{s}$, by SSP-PT method $\Delta \tau_{S S P-P T}=1.87 \mu s$ and by SSP-MPSD method $\Delta \tau_{S S P}$ ${ }_{M P S D}=1.91 \mu \mathrm{s}$, corresponding to the detection error $1.1 \%$, $2.2 \%$ and $0 \%$, respectively. The average error is about $1 \%$.

Fig. 6 (c) shows the measured ultrasonic signal on specimen (A) in the undamaged zone with $5 \mathrm{Mhz}$ transducer. Fig. 6 (d) shows the TDOA results obtained by deconvolution $\Delta \tau_{\text {deconv }}=1.89 \mu \mathrm{s}$, by SSP-PT method $\Delta \tau_{S S P-P T}=1.68 \mu \mathrm{s}$ and by SSP-MPSD method $\Delta \tau_{S S P-M P S D}=$ $1.89 \mu \mathrm{s}$, corresponding to the detection error $1.1 \%, 12 \%$
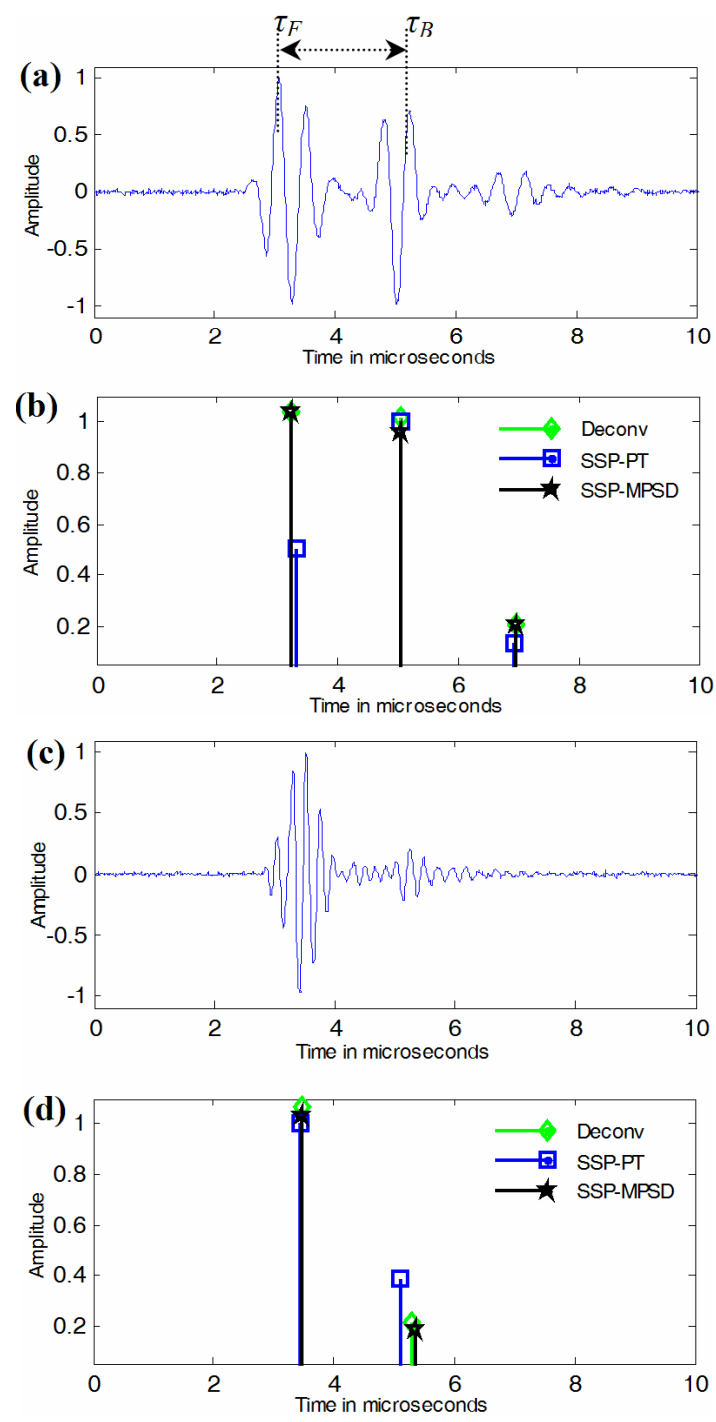

Fig. 6. Experimental signals of specimen(A) in undamaged zone, (a) with $2.25 \mathrm{Mhz}$ transducer frequency, (b) Results obtained by deconvolution, SSP-PT and SSP-MPSD ( $Q=5$, filters number $=10$ and $f_{1}=0.9$ $\mathrm{Mhz}$, (c) with $5 \mathrm{Mhz}$ transducer frequency, (d) Results obtained by deconvolution, SSP-PT and $\operatorname{SSP}-\operatorname{MPSD}\left(Q=5\right.$, filters number $=10$ and $f_{l}=2.45$ Mhz) 
and $1 \%$, respectively. The average error is about $4.7 \%$. It is important to note that here (i.e., in the case of $5 \mathrm{Mhz}$ transducer) the SSP-PT gives a high error compared to the
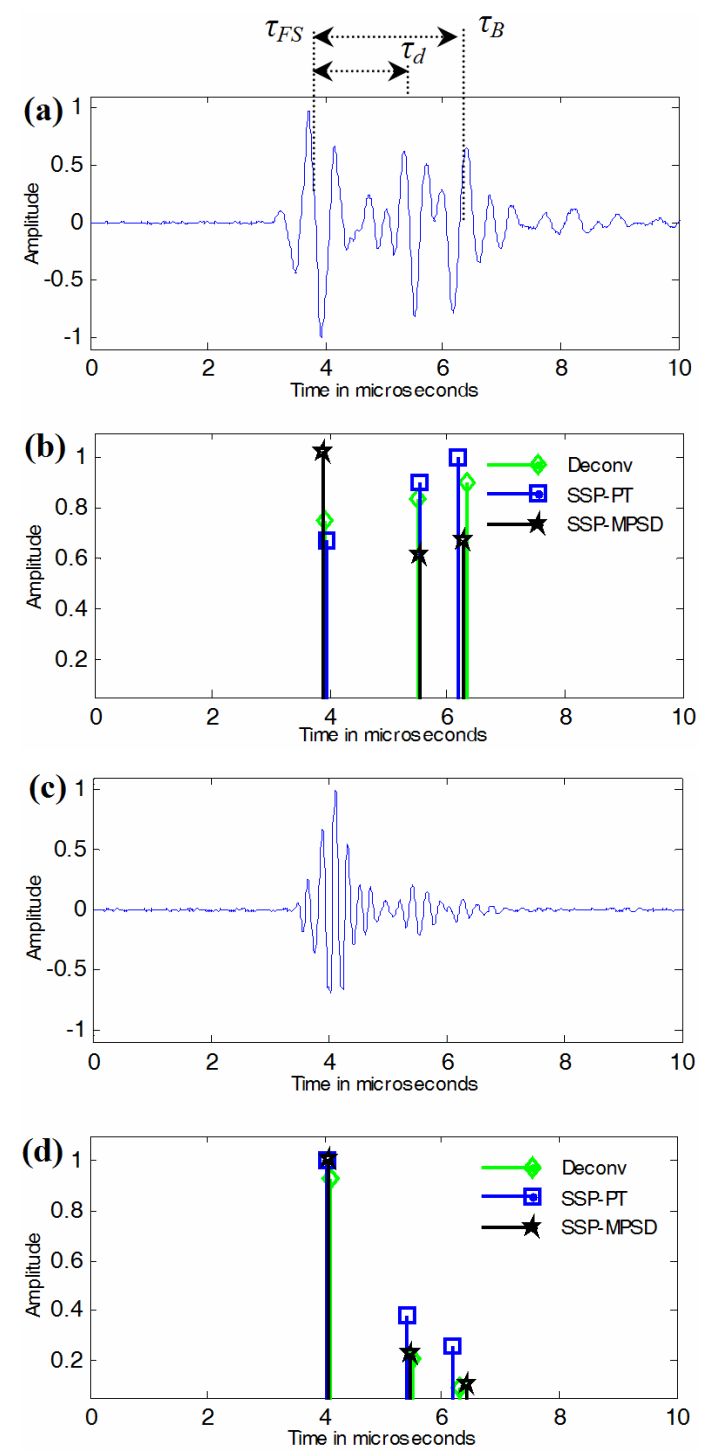

Fig. 7. Experimental signals of specimen (A) in damaged zone, (a) with $2.25 \mathrm{Mhz}$ transducer frequency, (b) Results of (a) obtained by deconvolution, SSP-PT and SSP-MPSD $\left(Q=5\right.$, filters number $=9$ and $f_{l}=$ $1.4 \mathrm{Mhz}$ ), (c) with $5 \mathrm{Mhz}$ transducer frequency, (d) Results of (c) obtained by deconvolution, SSP-PT and SSP-MPSD $\left(Q=5\right.$, filters number $=10$ and $f_{l}=$ 2.45Mhz) deconvolution and SSP-MPSD.

Fig. 7 (a) shows the measured echoes of the CFRP specimen (A) in damaged zone with $2.25 \mathrm{Mhz}$ transducer. Three echoes can be found in the signal: The first echo represents the front surface echo; the second echo represents the defect echo location of the delamination zone and the third echo represents the first back wall echo. After estimation by all methods (see Fig. 7 (b)), the delamination defect is detected by deconvolution method at a depth of $\tau_{d-}$ deconv $=1.55 \mu \mathrm{s}$, by SSP-PT method at a depth of $\tau_{d-S S P}$ ${ }_{P T}=1.59 \mu \mathrm{s}$ and by SSP-MPSD method at a depth of $\tau_{d-S S P}$. $M P S D=1.67 \mu \mathrm{s}$.

Fig. 7 (c) shows the measured ultrasonic signal on specimen (A) in damaged zone with $5 \mathrm{Mhz}$ transducer. Fig. 7 (d) shows the results, the delamination defect is detected by deconvolution method at a depth of $\tau_{d \text {-deconv }}=$ $1.41 \mu \mathrm{s}$, by SSP-PT method at a depth of $\tau_{d-S S P-P T}=1.37 \mu \mathrm{s}$ and by SSP-MPSD method at a depth of $\tau_{d-S S P-M P S D}=1.39 \mu \mathrm{s}$.

In the case of delamination defect with $2.25 \mathrm{Mhz}$ transducer, the average error is about 3\% compared to the average error of $10 \%$ in the case of $5 \mathrm{Mhz}$ transducer.

The obtained results on CFRP Specimen (A) with the three methods are tabulated in Table 1 in terms of the time difference of arrival (TDOA), the thickness of the specimen, the position of the delamination, and the error in $\%$.

We can note that the SSP-MPSD gives an average error of $1 \%$ in the case of thickness measurement. This result provides evidence of the good performance of SSP-MPSD method which has improved accuracy in the estimation of echo arrival time.

The specimen (B) is made of eight layers joined with epoxy, one layer on the other altering the orientation from $\left(0^{\circ}, 45^{\circ}\right)$. This specimen is divided in two zones (see Fig. 8):

- The $1^{\text {st }}$ zone is an undamaged zone with $2.9 \mathrm{~mm}$ thickness.

- The $2^{\text {nd }}$ zone is a damaged zone. The delamination

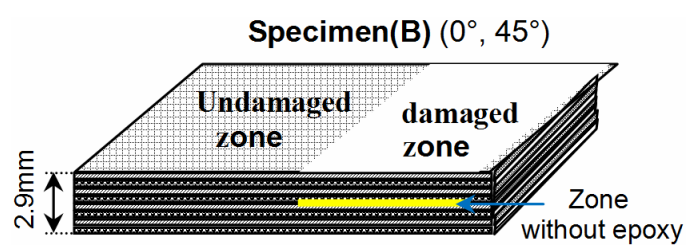

Fig. 8. Geometry and dimension of the CFRP specimen (B)

Table 1. Thickness of the specimen (A) and depth of defect with error in $\%$

\begin{tabular}{|c|c|c|c|c|c|c|c|c|}
\hline & & \multicolumn{3}{|c|}{$2.25 \mathrm{Mhz}$} & \multicolumn{3}{|c|}{$5 \mathrm{Mhz}$} & \multirow{2}{*}{$\begin{array}{l}\text { Real value } \\
\quad(\mathrm{mm})\end{array}$} \\
\hline & & Deconv & SSP-PT & SSP-MPSD & Deconv & SSP-PT & SSP-MPSD & \\
\hline $\begin{array}{c}\text { Undamaged } \\
\text { zone }\end{array}$ & $\begin{array}{l}\text { Thickness of the } \\
\text { specimen }\end{array}$ & $\begin{array}{c}2.67 \mathrm{~mm} \\
1.1 \%\end{array}$ & $\begin{array}{c}2.64 \mathrm{~mm} \\
2.22 \%\end{array}$ & $\begin{array}{c}2.7 \mathrm{~mm} \\
0 \%\end{array}$ & $\begin{array}{c}2.67 \mathrm{~mm} \\
1.1 \%\end{array}$ & $\begin{array}{c}2.37 \mathrm{~mm} \\
12 \%\end{array}$ & $\begin{array}{c}2.67 \mathrm{~mm} \\
1 \%\end{array}$ & $2.7 \mathrm{~mm}$ \\
\hline \multirow{2}{*}{$\begin{array}{l}\text { Damaged } \\
\text { zone }\end{array}$} & $\begin{array}{l}\text { Thickness of the } \\
\text { specimen }\end{array}$ & $\begin{array}{c}3.42 \mathrm{~mm} \\
3.6 \%\end{array}$ & $\begin{array}{c}3.15 \mathrm{~mm} \\
4.5 \%\end{array}$ & $\begin{array}{c}3.38 \mathrm{~mm} \\
2.4 \%\end{array}$ & $\begin{array}{c}3.14 \mathrm{~mm} \\
4.8 \%\end{array}$ & $\begin{array}{c}3.04 \mathrm{~mm} \\
7.8 \%\end{array}$ & $\begin{array}{c}3.33 \mathrm{~mm} \\
0.9 \%\end{array}$ & $3.3 \mathrm{~mm}$ \\
\hline & $\begin{array}{l}\text { Position of the } \\
\text { delamination }\end{array}$ & $\begin{array}{c}2.19 \mathrm{~mm} \\
0.4 \%\end{array}$ & $\begin{array}{c}2.24 \mathrm{~mm} \\
1.8 \%\end{array}$ & $\begin{array}{c}2.36 \mathrm{~mm} \\
7 \%\end{array}$ & $\begin{array}{c}1.99 \mathrm{~mm} \\
9 \%\end{array}$ & $\begin{array}{c}1.93 \mathrm{~mm} \\
12 \%\end{array}$ & $\begin{array}{c}1.96 \mathrm{~mm} \\
10 \%\end{array}$ & $2.2 \mathrm{~mm}$ \\
\hline
\end{tabular}


defect is a zone without epoxy situated in the middle of the specimen (i.e. between layers 4 and 5).

Fig. 9 (a) shows the measured echoes of the CFRP
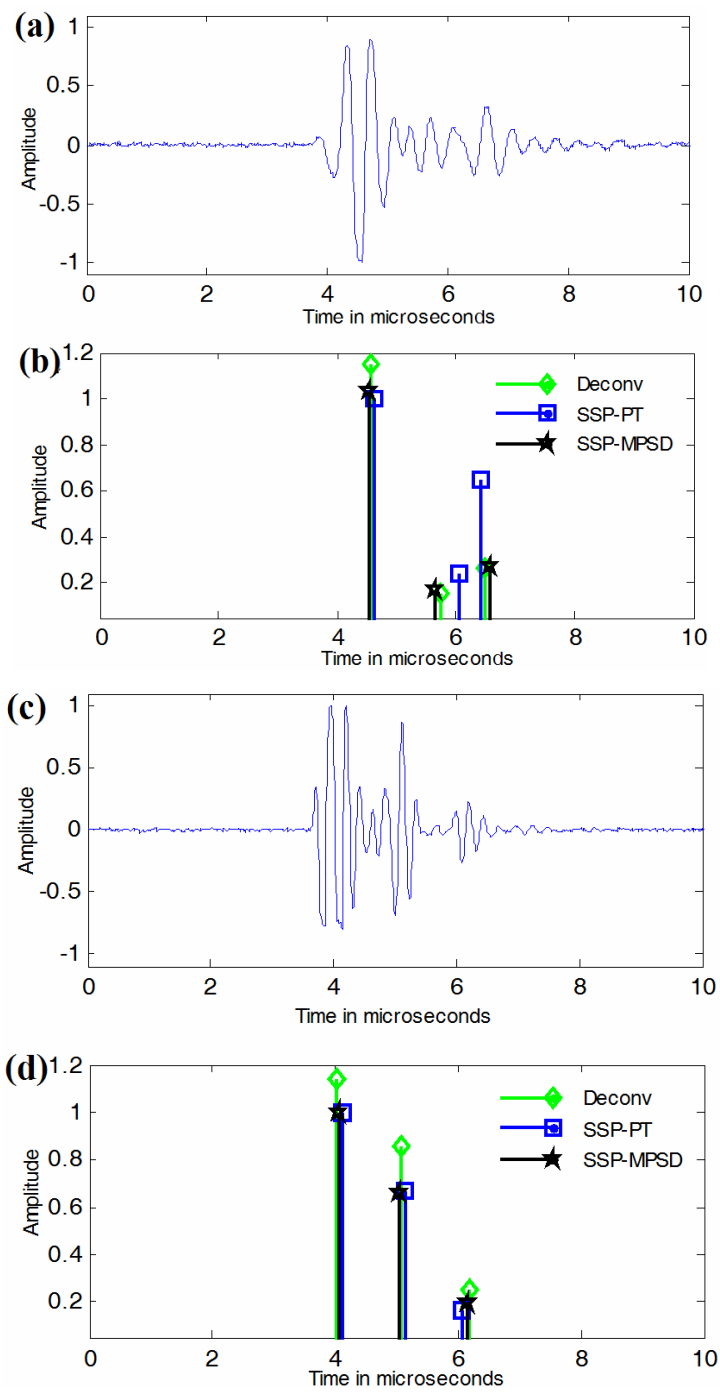

Fig. 9. Experimental signals of specimen (B) in damaged zone, (a) with $2.25 \mathrm{Mhz}$ transducer frequency, (b) Results of (a) obtained by deconvolution, SSP-PT and SSP-MPSD $\left(Q=5\right.$, filters number $=9$ and $f_{l}$ $=1 \mathrm{Mhz}$ ), (c) with $5 \mathrm{Mhz}$ transducer frequency, (d) Results obtained by deconvolution, SSP-PT and SSP-MPSD $\left(Q=5\right.$, filters number $=9$ and $f_{l}=$ 2.4 Mhz). specimen (B) in damaged zone with $2.25 \mathrm{Mhz}$ transducer. After estimation by all methods (see Fig. 9(b)), the delamination defect is detected by deconvolution method at a depth of $\tau_{\text {d-deconv }}=1.19 \mu \mathrm{s}$, by SSP-PT method at a depth of $\tau_{d-S S P-P T}=1.46 \mu \mathrm{s}$ and by SSP-MPSD method at a depth of $\tau_{d-}$ $S S P-M P S D=1.1 \mu \mathrm{s}$, corresponding to the detection error $12 \%$, $37 \%$ and $3 \%$, respectively.

Fig. 9 (c) shows the measured ultrasonic signal on specimen (B) in damaged zone with $5 \mathrm{Mhz}$ transducer. Fig. $9 \mathrm{~d}$ shows the results, the delamination defect is detected by deconvolution method at a depth of $\tau_{d \text {-deconv }}=1.05 \mu \mathrm{s}$, by SSP-PT method at a depth of $\tau_{d-S S P-P T}=1.03 \mu \mathrm{s}$ and by SSPMPSD method at a depth of $\tau_{d-S S P-M P S D}=1.07 \mu \mathrm{s}$, corresponding to the detection error $1.3 \%, 3 \%$ and $0.6 \%$, respectively.

We can note that the SSP-MPSD gives a better result, in the case of $2.25 \mathrm{Mhz}$ transducer the error is about $3 \%$ and $0.6 \%$ in the case of $5 \mathrm{Mhz}$ transducer.

The obtained results on CFRP Specimen (B) are recapitulated in Table 2.We can note that the SSP-MPSD gives an average error of $2 \%$ in the case of thickness measurement and detection of delamination.

The specimen $(\mathrm{C})$ is made of eight layers joined with epoxy, one layer on the other altering the orientation from $\left(0^{\circ}, 90^{\circ}\right)$. This specimen is divided in two zones (see Fig. 10):

- The $1^{\text {st }}$ zone is an undamaged zone with $3.1 \mathrm{~mm}$ thickness.

- The $2^{\text {nd }}$ zone is a damaged zone. The delamination defect is a nylon sheet, with $0.1 \mathrm{~mm}$ thickness situated in the middle of the part (i.e. between layers 4 and 5).

Fig. 11(a) shows the measured echoes of the CFRP specimen (C) in damaged zone with $2.25 \mathrm{Mhz}$ transducer. After estimation from all methods (see Fig. 11(b)), the

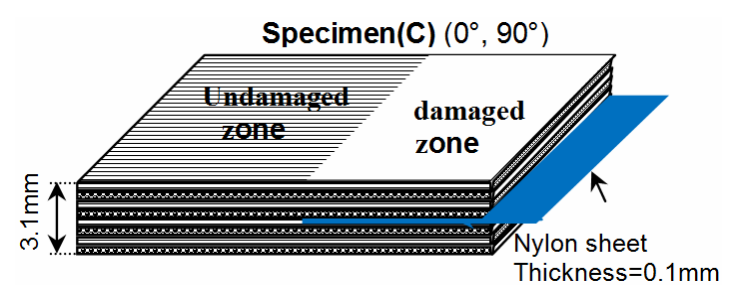

Fig. 10. Geometry and dimension of the CFRP specimen (C) (delamination defect is a nylon sheet situated between layers 4 and 5).

Table 2. Thickness of the specimen (B) and depth of defect with error in $\%$

\begin{tabular}{|c|c|c|c|c|c|c|c|c|}
\hline & & \multicolumn{3}{|c|}{$2.25 \mathrm{Mhz}$} & \multicolumn{3}{|c|}{$5 \mathrm{Mhz}$} & \multirow{2}{*}{$\begin{array}{c}\text { Real value } \\
(\mathrm{mm})\end{array}$} \\
\hline & & Deconv & SSP-PT & SSP-MPSD & Deconv & SSP-PT & SSP-MPSD & \\
\hline Undamaged zone & $\begin{array}{c}\text { Thickness of the } \\
\text { specimen }\end{array}$ & $\begin{array}{c}2.84 \mathrm{~mm} \\
2 \%\end{array}$ & $\begin{array}{c}2.51 \mathrm{~mm} \\
13 \%\end{array}$ & $\begin{array}{c}2.85 \mathrm{~mm} \\
1.7 \% \\
\end{array}$ & $\begin{array}{c}3.01 \mathrm{~mm} \\
3.7 \% \\
\end{array}$ & $\begin{array}{c}2.74 \mathrm{~mm} \\
5.5 \%\end{array}$ & $\begin{array}{c}2.99 \mathrm{~mm} \\
3 \% \\
\end{array}$ & $2.9 \mathrm{~mm}$ \\
\hline \multirow[b]{2}{*}{ Damaged zone } & $\begin{array}{c}\text { Thickness of the } \\
\text { specimen }\end{array}$ & $\begin{array}{c}2.73 \mathrm{~mm} \\
9 \%\end{array}$ & $\begin{array}{c}2.54 \mathrm{~mm} \\
15 \%\end{array}$ & $\begin{array}{c}2.88 \mathrm{~mm} \\
4 \%\end{array}$ & $\begin{array}{c}3.04 \mathrm{~mm} \\
1.3 \%\end{array}$ & $\begin{array}{c}2.78 \mathrm{~mm} \\
7 \%\end{array}$ & $\begin{array}{c}2.98 \mathrm{~mm} \\
0.6 \%\end{array}$ & $3 \mathrm{~mm}$ \\
\hline & $\begin{array}{l}\text { Position of the } \\
\text { delamination }\end{array}$ & $\begin{array}{c}1.68 \mathrm{~mm} \\
12 \%\end{array}$ & $\begin{array}{c}2.06 \mathrm{~mm} \\
37 \%\end{array}$ & $\begin{array}{c}1.55 \mathrm{~mm} \\
3 \%\end{array}$ & $\begin{array}{c}1.48 \mathrm{~mm} \\
1.3 \% \\
\end{array}$ & $\begin{array}{c}1.45 \mathrm{~mm} \\
3 \%\end{array}$ & $\begin{array}{c}1.51 \mathrm{~mm} \\
0.6 \%\end{array}$ & $1.5 \mathrm{~mm}$ \\
\hline
\end{tabular}


delamination defect is detected by deconvolution method at a depth of $\tau_{\text {d-deconv }}=1.17 \mu \mathrm{s}$, by SSP-PT method at a depth of $\tau_{d-S S P-P T}=1.27 \mu s$ and by SSP-MPSD method at a depth of $\tau_{d-}$ $S S P-M P S D=1.13 \mu \mathrm{s}$, corresponding to the detection error $6.4 \%$, $15 \%$ and $2.5 \%$, respectively.
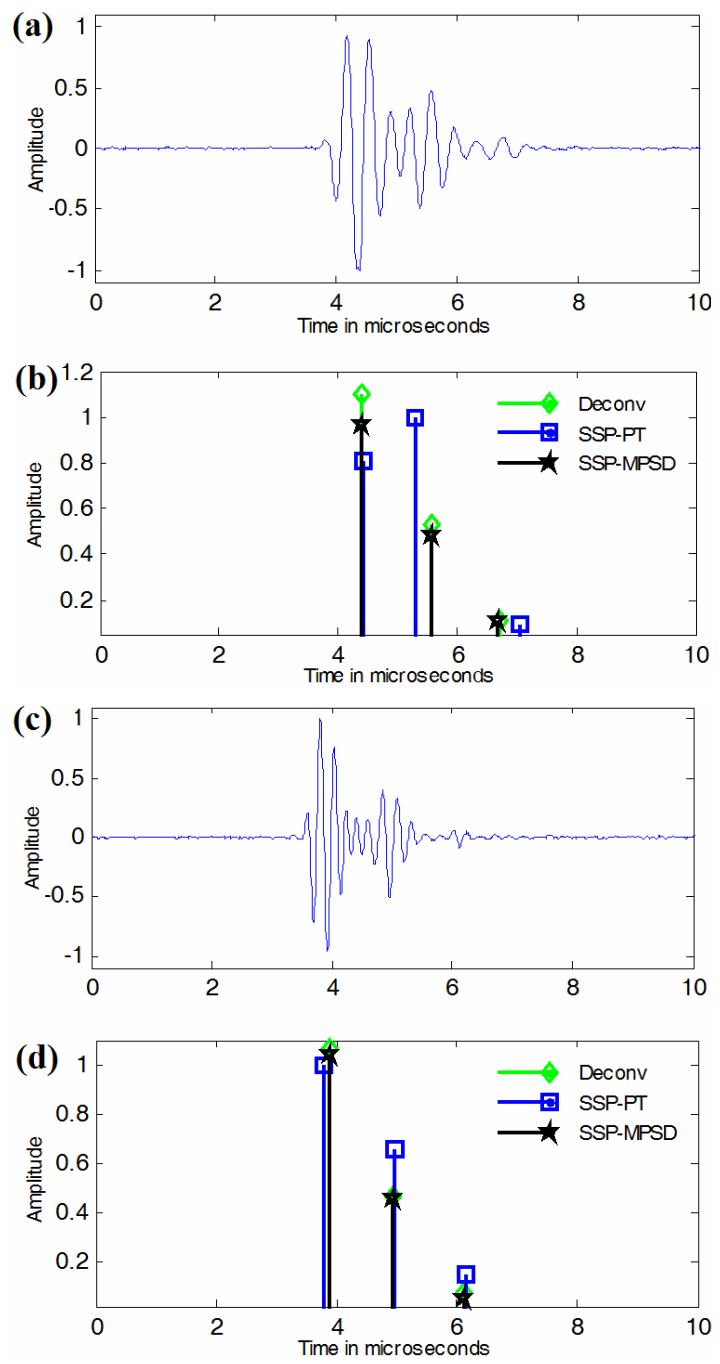

Fig. 11. Experimental signals of specimen (C) in damaged zone, (a) with $2.25 \mathrm{Mhz}$ transducer frequency, (b) Results of (a) obtained by deconvolution, SSP-PT and SSP-MPSD $\left(Q=5\right.$, filters number $=9$ and $f_{l}=$ $0.9 \mathrm{Mhz}$ ), (c) with $5 \mathrm{Mhz}$ transducer frequency, (d) Results obtained by deconvolution, SSP-PT and SSP-MPSD $\left(Q=5\right.$, filters number $=9$ and $f_{l}=2.4$ $\mathrm{Mhz}$ )
Fig. 11(c) shows the measured ultrasonic signal on specimen (C) in damaged zone with $5 \mathrm{Mhz}$ transducer. Fig. 11(d) shows the results, the delamination defect is detected by deconvolution method at a depth of $\tau_{d-\text { deconv }}=1.06 \mu \mathrm{s}$, by SSP-PT method at a depth of $\tau_{d-S S P-P T}=1.2 \mu s$ and by SSPMPSD method at a depth of $\tau_{d-S S P-M P S D}=1.06 \mu \mathrm{s}$. corresponding to the detection error $3.8 \%, 9 \%$ and $3.8 \%$, respectively.

We can note that the SSP-MPSD gives a better result, in the case of $2.25 \mathrm{Mhz}$ transducer, the error is about $2.5 \%$.

The obtained results on CFRP Specimen (C) are recapitulated in Table 3.

\section{Conclusions}

In this work, three types of delamination defects (Teflon, Air and Nylon) were inserted into carbon fiber reinforced polymer multi-layered composite materials. The tests were carried out by two broad band ultrasonic transducer of frequencies $2.25 \mathrm{Mhz}$ and $5 \mathrm{Mhz}$, with same quality factor. The aim of these tests is to check the performances of the algorithms (Deconvolution, SSP-PT and SSP-MPSD) in determining defects position and resolution of detected defect position.

In consideration of the obtained results (Tables 1,2 and 3 ); when the defect is close to the back face, increasing the frequency does not improve the resolution, since the error of localization by SSP-MPSD method passed from $7 \%$ to $10 \%$ for the frequencies of $2.25 \mathrm{Mhz}$ and $5 \mathrm{Mhz}$, respectively. This is explained by the attenuation of the materials which altered the quality of the obtained signals.

The comparison of the three types of defects gave the following results:

- The presence of a Teflon layer is precisely detected by the deconvolution $(0.4 \%)$.

- As for the layers of air or nylon in material, it is recommended to use SSP-MPSD which gives a more precise localization around $3 \%$. In the case of thickness measurement of the composite samples, we can note that SSP-PT method gives an average error of $7.76 \%$, the deconvolution gives an average error of $2.77 \%$ and SSP-MPSD method gives an average error of $1.33 \%$. The obtained results show that the error on the thickness measurement is lower with SSP-MPSD method. In conclusion, we can note that the SSPMPSD method can locate with accuracy the reflected echoes on CFRP composite materials.

Table 3. Thickness of the specimen (C) and depth of defect with error in \%

\begin{tabular}{|c|c|c|c|c|c|c|c|c|}
\hline & & \multicolumn{3}{|c|}{$2.25 \mathrm{Mhz}$} & \multicolumn{3}{|c|}{$5 \mathrm{Mhz}$} & \multirow{2}{*}{$\begin{array}{l}\text { Real value } \\
\text { (mm) }\end{array}$} \\
\hline & & Deconv & SSP-PT & SSP-MPSD & Deconv & SSP-PT & SSP-MPSD & \\
\hline $\begin{array}{l}\text { Undamaged } \\
\text { zone }\end{array}$ & $\begin{array}{l}\text { Thickness of the } \\
\text { specimen }\end{array}$ & $\begin{array}{c}3.18 \mathrm{~mm} \\
2.5 \%\end{array}$ & $\begin{array}{c}3.24 \mathrm{~mm} \\
4.5 \%\end{array}$ & $\begin{array}{c}3.11 \mathrm{~mm} \\
0.3 \%\end{array}$ & $\begin{array}{c}3.08 \mathrm{~mm} \\
0.6 \%\end{array}$ & $\begin{array}{c}3.15 \mathrm{~mm} \\
1.6 \%\end{array}$ & $\begin{array}{c}3.08 \mathrm{~mm} \\
0.6 \%\end{array}$ & $3.1 \mathrm{~mm}$ \\
\hline \multirow{2}{*}{$\begin{array}{l}\text { Damaged } \\
\text { zone }\end{array}$} & $\begin{array}{l}\text { Thickness of the } \\
\text { specimen }\end{array}$ & $\begin{array}{c}3.26 \mathrm{~mm} \\
1.8 \%\end{array}$ & $\begin{array}{c}3.72 \mathrm{~mm} \\
16 \%\end{array}$ & $\begin{array}{c}3.21 \mathrm{~mm} \\
0.3 \%\end{array}$ & $\begin{array}{c}3.14 \mathrm{~mm} \\
1.8 \%\end{array}$ & $\begin{array}{c}3.33 \mathrm{~mm} \\
4 \%\end{array}$ & $\begin{array}{c}3.16 \mathrm{~mm} \\
1.25 \%\end{array}$ & $3.2 \mathrm{~mm}$ \\
\hline & $\begin{array}{c}\text { Position of the } \\
\text { delamination }\end{array}$ & $\begin{array}{c}1.65 \mathrm{~mm} \\
6.4 \%\end{array}$ & $\begin{array}{c}1.79 \mathrm{~mm} \\
15 \%\end{array}$ & $\begin{array}{c}1.59 \mathrm{~mm} \\
2.5 \%\end{array}$ & $\begin{array}{c}1.49 \mathrm{~mm} \\
3.8 \%\end{array}$ & $\begin{array}{c}1.69 \mathrm{~mm} \\
9 \%\end{array}$ & $\begin{array}{c}1.49 \mathrm{~mm} \\
3.8 \%\end{array}$ & $1.55 \mathrm{~mm}$ \\
\hline
\end{tabular}




\section{References}

[1] R.S. Edwards, S. Dixon, X. Jian, "Characterisation of defects in the railhead using ultrasonic surface waves", NDT\&E Int. 2006 (39) 468-475.

[2] T. D’Orazio, M. Leo, A. Distante, C. Guaragnella, V. Pianese, G. Cavaccini, "Automatic ultrasonic inspection for internal defect detection in composite materials", NDT\&E Int. 2008, (41) 145-154.

[3] V. L. Newhouse, N. M. Bilgutay, J. Saniie, E. S. Furgason, "Flaw-to-gain echo enhancement by split spectrum processing". Ultrasonics, 1982, (20) 59-68.

[4] S.C. Ng, N. Ismail, Aidy Ali, Barkawi Sahari, J.M. Yusof, B.W. Chu, Non-destructive Inspection of Multi-layered Composites Using Ultrasonic Signal Processing, Materials Science and Engineering 2011, (17).

[5] H. C. Sun, J. Saniie, "Ultrasonic flaw detection using split spectrum processing combined with adaptivenetwork-based fuzzy inference systems", Proc IEEE Ultrason Sympos, 1999.

[6] R. Drai, A. Benammar and A. Benchaala, "Signal processing for the detection of multiple imperfection echoes drowned in the structural noise", Ultrasonics, 2004, (42), pp. 831-835.

[7] A. Benammar, R. Drai and A. Guessoum, "Detection of delamination defects in CFRP materials using ultrasonic signal processing", Ultrasonics, 2008, (48), pp. 731-738.

[8] M. Kesharaju, R. Nagarajah, T. Zhang, I. Crouch, Ultrasonic sensor based defect detection and characterisation of ceramics, Ultrasonics, January 2014, (54)(1), pp.312-317.

[9] S. Legendre, J. Goyette, D. Massicotte, "Ultrasonic NDE of composite material structures using wavelet coefficients", NDT\& E Int, 2001, (34)(1), pp. 31-37.

[10] H. Jeong, "Analysis of plate wave propagation in anisotropic laminates using a wavelet transform", NDT\& E Int, 2001, (34)(3), pp. 185-190.

[11] R. Drai, M. Khelil, A. Benchaala, "Time frequency and wavelet transform applied to selected problems in ultrasonics NDE", NDT\& E Int, 2002, (35), pp. 567-572.

[12] Y. Ding, R. Reuben, J. Steel, "A new method for waveform analysis for estimated $\mathrm{AE}$ wave arrival times using wavelet decomposition", NDT\& E Int, 2004, (37)(4), pp. 279-290.

[13] R. Piotrkowski, A. Gallego, M. García-Hernandez and J. Ruzzante, "Ti and Cr nitride coating / steel adherence assessed by acoustic emission wavelet analysis", NDT\& E Int, 2005, (38)(4), pp. 260-267.

[14] P. L. Yeh, P. L. Liu, "Application of the wavelet transform and the enhanced Fourier spectrum in the impact echo test", NDT\& E Int, 2008, (41), pp. 382394.

[15] V. Matz, R. Smid, S. Starman, M. Kreidl, "Signal-to- noise ratio enhancement based on wavelet filtering in ultrasonic testing", Ultrasonics, 2009, (49), pp. 752759.

[16] A. White, J.W. Hong, S. Hong, J.Choi, "Parameter estimation for wavelet transformed ultrasonic signals", NDT \& E Int, 2011, (44), pp. 32-40.

[17] G. Zhang, C. Zhang, D. M. Harvey, Sparse signal representation and its applications in ultrasonic NDE, Ultrasonics, March 2012, (52)(3), pp.351-363.

[18] N. Ruiz-Reyes, P. Vera-Candeas, J. Curpian-Alonso, J.C. Cuevas-Martıanez, J.L. Blanco-Claraco, "Highresolution pursuit for detecting flaw echoes close to the material surface in ultrasonic NDT", NDT\&E Int, 2006, (39), pp. 487-492.

[19] Y. Lu, G. Cardoso, R. Demirli and J. Saniie, "Chirplet transform for ultrasonic signal analysis and NDE applications", IEEE Ultrasonic Symposium Proceedings, 2005, pp. 536-539.

[20] Y. Lu, R. Demirli, and J. A. Saniie. "Comparative Study of Echo Estimation Techniques for Ultrasonic NDE Applications", IEEE Ultrasonics Symposium, 2006.

[21] P. Karpur, P.M. Shankar, J.L. Rose, V.L. Newhouse, "Split spectrum processing: determination of the available bandwidth for spectral splitting", Ultrasonics, 1988, (26), pp. 204-209.

[22] J. Xin, "Detection and resolution of multiple targets using time-frequency and deconvolution techniques", Ph.D. Thesis, Drexel University, June 1994.

[23] J-D. Aussel, "Split-spectrum processing with finite impulse response filters of constant frequency-toband width ratio", Ultrasonics, (28), 1990.

[24] G. Stephane. Mallat. Zhifeng Zhang. "Matching pursuits with time frequency dictionaries", IEEE Transactions on Signal Processing, 1993, (41)(12), pp. 3397-3415.

[25] S. S. Chen, D. L. Donoho, M.A. Saunders, "Atomic decomposition by basis pursuit", Technical report, Statistics department, Stanford university 1995.

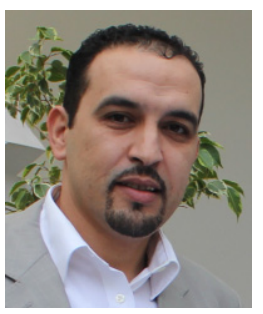

BENAMMAR Abdessalem Was born in Bouira, Algeria in 1976. He received his diploma of Engineering in Electronic from University of Blida, Algeria in 2000, he received his diploma of Magister in Electronic from University of Blida, Algeria in 2004, He received his PHD in Electronic from University of Blida, Algeria in 2010. He is head of department of monitoring and support of scientific and technological activities in welding and control. His research are based on signal and image processing applied to ultrasonic phased array and guided waves. 


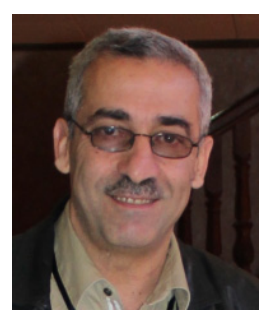

DRAI Radouane $\mathrm{He}$ received his doctor diploma in Electronic at Algiers University (USTHB). He is a director of research in Welding and NDT Research Centre CSC, and head of Signal and Image Processing Laboratory since 1986. His research works are based on ultrasonic and X-ray tomography. signal and image processing applied to 